\section{Ageing of higher organisms}

Principles of Mammalian Ageing. Second edition. By R. R. Kohn. Pp. 240. (Prentice-Hall: Englewood Cliffs, New Jersey, and Hemel Hempstead, UK, 1978.) \$19; £13.85.

Gerontology was born in optimism. It threatens to founder in verbiage. Papers and books are now appearing in quantity, while declining in quality, drowning the few really important studies in this field. Robert Kohn's book was there at the beginning of the boom, and it stands aside from the information explosion. Despite certain problems, this new edition restores to us a compact, well-written and refreshingly intelligent introductory text on the ageing of higher organisms.

The book begins by exploring the progressive crystallisation and toughening of inorganic materials as an analogy to illustrate ageing. It is an analogy which some may find weak and misleading, for ageing is manifest by increasing disorder rather than order. Nevertheless it serves the author's purpose, for he moves on to an excellent summary of the ageing phenomena in the extracellular materials of the body. The following sections deal with ageing within cells, briefly covering the evidence for age-related changes in the dynamic renewal of cellular constituents and for long-term biochemical deterioration. This is followed by a carefully ordered chapter on ageing of cell populations, which classifies cells according to their degree of continuing cell division within tissues. The rest of the book is concerned with the ageing of animals and here the author makes a good job out of the onerous task of summarising a highly complex and contradictory field.

The problem with the book is the author's penchant for selecting two or three seemingly contradictory papers as representative of major fields of ageing research, which then allows him to conclude that this work is inconclusive. For example, there is excellent evidence that dividing cell populations such as mammary gland cells, antibody-producing cells or haematopoietic cells show some age changes during the lifespan of the individual and will eventually cease division, although they are capable of outliving the organism from which they are derived. The author fails to mention significant work in this field and uses sparse studies to support a 'no-evidence' conclusion. He does the same with the exciting new field of the analysis of errors in macromolecules of senescent cells, with work on histones, on the question of protein turnover, repair capacity, DNA synthesis and the genetics of ageing as a whole. A more penetrating analysis would have shown him that work which at first reading seems contradictory is in fact complementary, and would have enabled him to pick out the key papers in each field. This would have allowed him to draw constructive conclusions in each area, thus enriching the book.

The author's unwillingness to make conclusions derives in part from a certain failure to properly grasp molecular, genetic and evolutionary concepts as a whole. He remakes the classic faux pas that ageing exists because "old, non-reproductive animals compete with younger animals for food", which is the same as saying that ageing exists because of ageing. Besides this, scientists usually have some inner vision which guides their work, especially in such a complex and

\section{Transfer RNAs}

Transfer RNA. Edited by S. Altman. Pp. 356. (MIT Press : Cambridge, Massachusetts, and London, 1979.) \$30; £21.

THE concept of tRNAs as the essential links between nucleic acid and protein, has for over twenty years been a central theme of molecular biology in general and studies on protein biosynthesis in particular. It is now some thirteen years since the 'state of the art' was comprehensively reviewed in a Cold Spring Harbor Symposium volume. Subsequent years have witnessed a veritable explosion in the literature, not only in classical areas of tRNA research but also in new ones such as analysis of tRNA genes, tRNAs as primers of reverse transcriptase activity, and determination of tRNA tertiary structure.

It is thus more than timely for an overview of all these aspects of tRNA function. This book succeeds admirably in this task, by providing a number of reviews, all by leading authorities in the field. The introduction (by Zachau) serves to establish the subject in a historical perspective, as well as reminding us of the many questions still left unanswered. The impact of our knowledge of the tertiary structure of just one tRNA is well illustrated throughout the book, and not only by the chapter on its crystal structure and immediate implications (by Kim). In particular, Clark's chapter collating features of primary, secondary and tertiary structure provides a fascinating account of the generalisations and clues to function at the molecular level provided by the yeast phenylalanine tRNA crystal exploratory field as ageing research. To some, ageing is a process of headlong over-differentiation. To others it is an ineluctable entropic deterioration of biological information. Krohn seems to see ageing as a kind of crystallisation or fossilisation of biological materials. Each man to his own. But it is important to be able to understand other researchers' ways of looking at the problem.

Yet it is easy to criticise a scientific text on the basis of selectivity. These criticisms do not alter the fact that the book hoids together very well. Now that the number of papers on ageing is somewhere at the five figure level, a coherent and thoughtful introductory text such as this is no mean feat.

Stephen J. Fulder

Stephen J. Fulder is about to take up a research post at the Brookdale Institute of Gerontology, Jerusalem, Israel.

structure. The essay by Crothers and Cole on conformational changes of tRNA in solution serves to provide a physically based viewpoint complementary to descriptions of structure in the crystalline state.

The contribution of Iglio and Cramer on tRNA synthetases and their substrate interactions, elegantly describes the current status in this area, to which Cramer's group have themselves made many significant contributions. The beginnings of understanding at the molecular level are also discussed by Pongs in a chapter largely concerned with ribosomal and $\mathrm{mRNA}$ recognition in relation to protein synthesis. Both these chapters clearly indicate the important directions to pursue in the future, difficult though they will be.

Other sections in this book are more concerned with biological topics. Thus, Altman himself reviews the area of tRNA biosynthesis, and with Körner and Feinstein he discusses tRNAmediated suppression. Many other roles of TRNA are reviewed by LaRossa and Söll, Nishimura, in a chapter on modified nucleosides and isoaccepting tRNAs, suggests that many of these regulatory roles involve modified residues, in as yet unrevealed ways.

Throughout this book the sense of excitement still purveying many areas of transfer RNA study, come across very clearly. It can thus be recommended as a worthwhile and stimulating read for graduate and even advanced undergraduate students in molecular biology as well as for those workers more actively involved in the subject.

Stephen Neidle

Stephen Neidle works in the Department of Biophysics, King's College, University of London, UK. 